\title{
One-Point Microwave Radiometer Calibration
}

\author{
Ignasi Corbella $^{\circledR}$, Francesc Torres, Senior Member, IEEE, Josep Closa, Nuria Duffo, Senior Member, IEEE, \\ Israel Durán, Verónica González-Gambau ${ }^{\circledR}$, and Manuel Martín-Neira ${ }^{\circledR}$, Senior Member, IEEE
}

\begin{abstract}
A method for internally calibrating microwave total power radiometers by using only one level of noise injection is presented. It is based on having a previous accurate characterization of the receiver noise temperature, which used de facto as a second calibration standard. The method proves to be at least equivalent to the classical two level, as demonstrated through their intercomparison using the data provided by the Microwave Imaging Radiometer using Aperture Synthesis (MIRAS) on board the European Space Agency Soil Moisture and Ocean Salinity (SMOS) Satellite. The long-term stability in terms of retrieved brightness temperature using both methods has similar trends with a small advantage for the one-point approach proposed here.
\end{abstract}

Index Terms-Calibration, microwave radiometry.

\section{INTRODUCTION}

$\mathbf{M}$ ICROWAVE radiometers are highly sensitive receivers used to measure natural thermal emission and infer from it the physical properties of the emitting body. They have been used for decades in applications ranging from earth observation to radio astronomy or even planetary sensing. Accurate radiometer calibration is fundamental to get the required information out of the output voltages or counts provided by the receivers.

In the microwave region, the Rayleigh-Jeans law applies so thermal emission is characterized by a brightness temperature, defined as the emissivity times the physical temperature. The power collected by a radiometer antenna is then characterized by the antenna noise temperature. In this context, radiometer calibration is understood as the process to estimating the antenna noise temperature out of the receiver's output signal.

Assuming a linear relationship between both, the calibration is reduced to estimating two parameters: a slope and a bias.

Manuscript received February 25, 2019; revised May 13, 2019; accepted June 10, 2019. Date of publication July 1, 2019; date of current version February 26, 2020. This work was supported in part by the European Space Agency with Deimos Enginheria (Portugal) under SMOS P7 Subcontract DME CP12 2015-005 and in part by the Ministerio de Economía, Industria y Competitividad, Gobierno de España under Project TEC2014-58582-R, Project TEC2017-88850-R, and Project ESP2015-67549C3-1-R. (Corresponding author: Ignasi Corbella.)

I. Corbella, F. Torres, N. Duffo, and I. Durán are with the Remote Sensing Laboratory, Universitat Politècnica de Catalunya, 08034 Barcelona, Spain (e-mail: corbella@tsc.upc.edu; xtorres@tsc.upc.edu; duffo@tsc.upc.edu; israel.duran@tsc.upc.edu).

J. Closa is with Airbus Defence and Space SAU, 28022 Madrid, Spain (e-mail: josep.closa@airbus.com).

V. González-Gambau is with the Department of Physical Oceanography, Institute of Marine Sciences (ICM), CSIC and Barcelona Expert Center, 08003 Barcelona, Spain (e-mail: vgonzalez@icm.csic.es).

M. Martín-Neira is with the European Space Research and Technology Centre, European Space Agency, 2200 AG Noordwijk, The Netherlands (e-mail: manuel.martin-neira@esa.int).

Color versions of one or more of the figures in this letter are available online at http://ieeexplore.iee.org.

Digital Object Identifier 10.1109/LGRS.2019.2923539

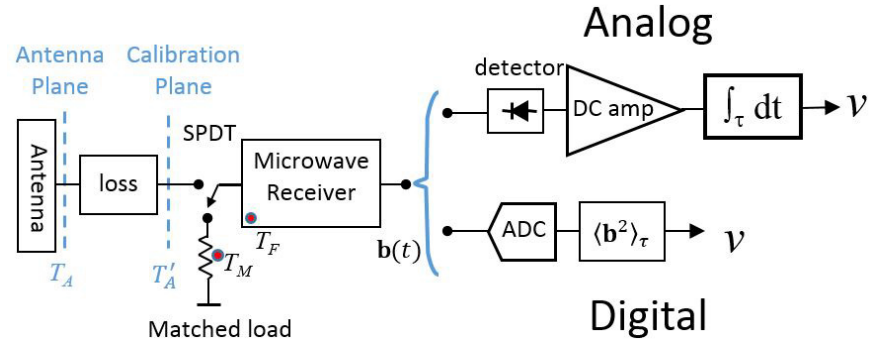

Fig. 1. Simplified block diagram of a microwave radiometer using onepoint internal calibration. Digital and analog versions are shown. Red dots: temperature sensors.

The classical approach consists of measuring two known standards and solve both unknowns from the corresponding measurements [1]. The commonly used calibration loads are noise diodes, matched loads at known temperatures, ambient or cryogenic, or active cold loads [2], [3]. In all cases, the calibration mechanism is the same and its quality depends on the accurate knowledge of the calibration standards' noise temperature. They have, thus, to be previously characterized on a controlled environment before their use in scientific measurements. Any unexpected or uncontrolled change in the calibration standards induces necessarily a calibration error.

During the radiometer operation, switches located near the antenna are used to measure alternatively power coming from the antenna and from the calibration loads. Time dedicated to calibrate is always at the expense of science measurements, so it is, in general, advisable to minimize the calibration frequency without compromising the measurement quality and stability.

This letter presents an internal calibration approach that requires only one load to periodically switch at the receiver's input. It is based on having an accurate receiver characterization and is implemented as an alternative method on the Microwave Imaging Radiometer using Aperture Synthesis (MIRAS) instrument on board the Soil Moisture and Ocean Salinity (SMOS) Mission [4].

\section{One-Point CALIBRATION}

The block diagram of a single-channel radiometer using the proposed one-point calibration is drawn in Fig. 1. A single pole double throw (SPDT) switch is used to measure either the power coming from the antenna or a matched load at known (ambient) temperature $T_{M}$. The signal is then amplified and filtered by a low noise microwave receiver that may or not include frequency conversion. In an analog system, the output signal is detected by a diode to produce a dc voltage proportional to the output power. This voltage is amplified 


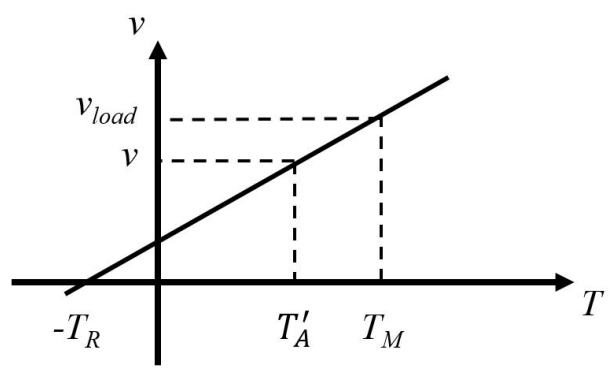

Fig. 2. Interpretation of the one-point calibration using the linear inputoutput radiometer response.

and integrated (filtered) during the required integration time. Detection, dc amplification, and filtering may originate a constant voltage added to the measurements independently of the input signal power. This "instrumental offset" can be measured using the four-point technique [5], which does not need known standards, and must be removed as a first calibration step. On the other hand, for the fully digital version (Fig. 1), the RF (or IF) signal is directly converted to digital counts and its power numerically computed. In this case, there is no instrumental offset.

In any case, assuming no (or canceled) instrumental offset, the output voltage becomes proportional to the total radiometric noise power and can be expressed as

$$
v=G\left(T_{A}^{\prime}+T_{R}\right)
$$

where $T_{A}^{\prime}$ is the antenna noise temperature referenced at the calibration plane, $G$ is the receiver's transducer power gain, and $T_{R}$ its noise temperature. Internal calibration aims at estimating both parameters in order to solve this equation for $T_{A}^{\prime}$

$$
T_{A}^{\prime}=\frac{v}{G}-T_{R} .
$$

To get the final antenna noise temperature $T_{A}$, all components between calibration and antenna planes must be accounted for by assuming known insertion loss and physical temperatures. This is a quite standard procedure not detailed here, but it is critical to get the most accurate radiometric measurements [2].

If the physical temperature of the switch is the same as that of the matched load, the output power when the switch connects the load, $v_{\text {load }}$, is given by (1) after substituting $T_{A}^{\prime}$ by $T_{M}$, the physical temperature of the load in K (Fig. 1). The gain can then be solved from (1) as

$$
G=\frac{v_{\text {load }}}{T_{M}+T_{R}} .
$$

Thus, if $T_{R}$ is known the gain can be estimated without the need of any additional measurement of a second calibration standard. Fig. 2 provides a graphical interpretation: the method actually uses two points, as required for solving a linear system with two unknowns, but one of them is the extrapolation of the linear input-output line to the ordinates origin. In other words, the receiver noise temperature $T_{R}$ becomes in this approach a calibration standard and must be accurately and independently measured.
Any uncertainty on the receiver noise temperature, either due to unexpected drifts or poor characterization, is obviously translated into an error in the calibrated gain. From (3), the relative gain and noise temperature errors relate to each other by

$$
\frac{\Delta G}{G}=-\frac{\Delta T_{R}}{T_{R}} \frac{T_{R}}{T_{M}+T_{R}}
$$

so gain errors are always attenuated with respect of those of $T_{R}$ (note that $T_{R} /\left(T_{M}+T_{R}\right)<1$ ).

As can be deduced from the graphical representation in Fig. 2, the closer the antenna temperature to the load physical temperature is, the lower is the error. This statement can be quantified by considering a perfectly stable receiver. Introducing (3) into (2) and assuming that the gain has not changed, it is found that

$$
T_{A}^{\prime}=\left(\frac{v}{v_{\text {load }}}-1\right) T_{R}+\frac{v}{v_{\text {load }}} T_{M} .
$$

The sensitivity of the measurement to any variation of $T_{R}$ is then

$$
\frac{\partial T_{A}^{\prime}}{\partial T_{R}}=\frac{v}{v_{\text {load }}}-1=\frac{T_{A}^{\prime}+T_{R}}{T_{M}+T_{R}}-1
$$

demonstrating that when $T_{A}^{\prime}$ approaches $T_{M}$, the measurement error tends to vanish.

The receiver noise temperature at calibration plane used in both (2) and (3) depends on physical temperature and can be expressed as

$$
T_{R}=T_{R}\left(T_{0}\right)+\operatorname{STR}\left(T_{F}-T_{0}\right)
$$

where $T_{F}$ is the temperature readout of the sensor located at the front end (see Fig. 1), $T_{R}\left(T_{0}\right)$ is the receiver noise temperature at a reference temperature $T_{0}$ and STR a sensitivity coefficient. All three parameters, namely, $T_{R}\left(T_{0}\right)$, STR, and $T_{0}$, are read from an external database produced in advance after a careful characterization of the receivers in a thermal chamber. Receiver noise temperature measurement is a highly standardized procedure that can provide low systematic uncertainties. Different matching conditions in the two states of the SPDT can be taken into account in this characterization.

Other internal calibration approaches that use two levels of noise injection are also based on calibration standard precharacterization (see [2]), so the need of receiver noise temperature characterization in the present approach is a similar solution. There is no reason to assume that the absolute noise temperature of a calibration load (a noise diode or an active cold load), with the corresponding transmission lines to reach the receiver's input, can be better characterized than the noise temperature of an amplifier.

\section{MIRAS EXAMPLE}

The instrument MIRAS on board SMOS satellite has 69 small antennas connected to corresponding microwave receivers, each one provided with an analog detector [4]. They conform 69 total power radiometers used to measure the visibility function amplitude [6]. They are calibrated using a standard two-point (hot/warm) schema by means of noise 

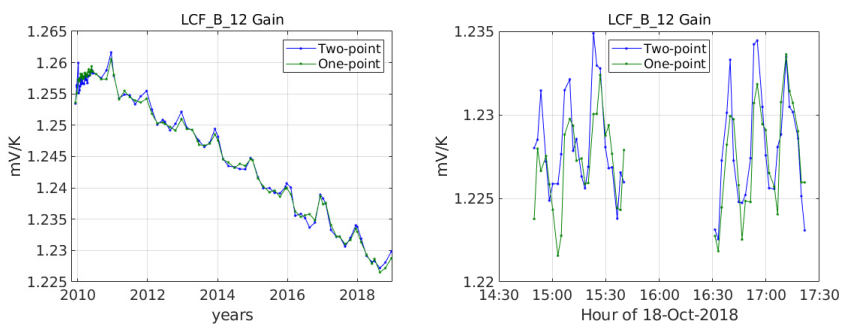

Fig. 3. Example of one-point and two-point gains taken from SMOS flight data. Left: long-term data. Right: Short-term (orbital) data.

diodes and distribution networks [7]. In addition, in order to correct for visibility offsets, all receivers are periodically switched to matched loads so as to generate uncorrelated input signals. Taking advantage of this operation and the fact that all receivers were fully characterized on-ground in terms of noise temperature, the one-point calibration procedure was implemented in parallel with the nominal approach. Although, up until now, only the two points are operationally used, both methods have been systematically compared throughout the mission lifetime, demonstrating similar performances and even a slightly better stability of the one-point case [8]. As an example, Fig. 3 shows, for one particular receiver, the measured gain using both methods in a long-term scale along all the mission and also within a calibration orbit. Their consistency is very high and shows that the one-point approach is at least as effective as the classical one in tracking gain variations and trends. Results for all 69 receivers are similar.

The quality of calibration can be better assessed by analyzing the temporal stability of the measured brightness temperature as a function of time. Following the same procedure as in [8], a set of daily orbits from 2010 to 2016 over the mid-Pacific ocean are selected. For all of them, the brightness temperature at the top of atmosphere is retrieved by processing SMOS data up to level $1 \mathrm{~B}$ using the MIRAS testing software [9] and the result is compared against the predictions of a forward model. ${ }^{1}$ The model represents fairly well the expected instrument measurement and is being routinely used by the SMOS level 1 team to assess brightness temperature retrievals.

Fig. 4 shows the stability results corresponding to both internal calibration strategies, one and two points. In both cases, the processing is carried out using the so-called "All-LICEF" approach [10], so all measurements are consistently derived from the detected voltages of the individual receivers. The plots show the difference between measurement and model averaged in the latitudinal range $\left[-40^{\circ} 5^{\circ}\right]$ for each orbit as a function of time. As seen, both calibration approaches provide consistent results and similar long-term stability trends, demonstrating that calibration using one point is able to trap the gain variations as efficiently, slightly better actually, as the classical two-point method. The long-term slope computed from the plots is $6 \mathrm{mK} / y e a r$ in the case of one point and $-19 \mathrm{mK} / \mathrm{year}$ in the case of two-point, demonstrating that the

\footnotetext{
${ }^{1}$ The authors would like to thank Joseph Tenerelli (OceanDataLab, France) for providing the ocean forward model.
}

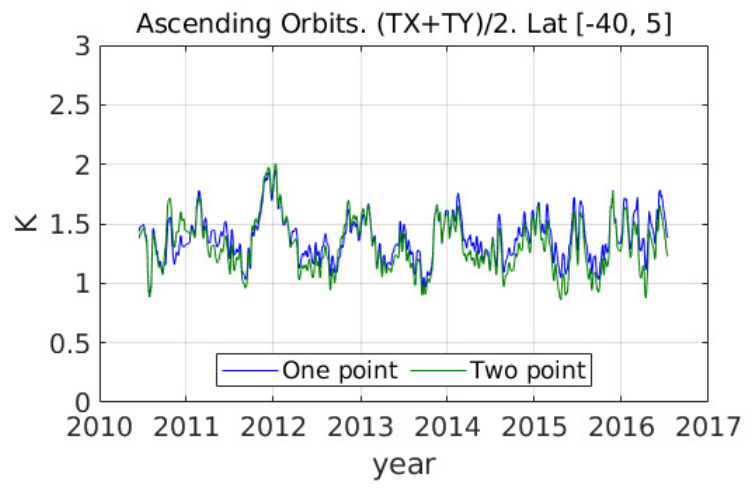

Fig. 4. Long-term Stability of SMOS brightness temperature for both calibration approaches. The plot represents the difference between the retrieved brightness temperature (average of both polarizations) and a forward model.

first case is able to track any gain variation even better than the second.

\section{CONCLUSION}

Total power microwave radiometers can be internally calibrated by switching the input port to a matched load at known ambient temperature, provided a good characterization of the receivers noise temperature as a function of physical temperature is available. Advantages with respect to the classical twolevel noise injection include, among others, front-end hardware simplification and reduction in overall calibration time. The results using the SMOS data show that the method is able to measure receivers' gain and their temporal variation as does the classical one. Brightness temperature retrievals using the one-point calibration show even better long-term stability with respect to using a hot/warm method. The method works as applied to MIRAS but needs to be evaluated for other radiometer systems.

\section{REFERENCES}

[1] F. T. Ulaby, R. K. Moore, and A. K. Fung, Microwave Remote Sensing. Active and Passive: Fundamentals and Radiometry, vol. 1. Norwood, MA, USA: Artech House, 1981.

[2] J. R. Piepmeier et al., "SMAP L-band microwave radiometer: Instrument design and first year on orbit," IEEE Trans. Geosci. Remote Sens., vol. 55, no. 4, pp. 1954-1966, Apr. 2017.

[3] S. T. Brown, S. Desai, W. Lu, and A. B. Tanner, "On the long-term stability of microwave radiometers using noise diodes for calibration," IEEE Trans. Geosci. Remote Sens., vol. 45, no. 7, pp. 1908-1920, May 2007.

[4] K. D. McMullan et al., "SMOS: The payload," IEEE Trans. Geosci. Remote Sens., vol. 46, no. 3, pp. 594-605, Mar. 2008.

[5] P. Piironen, "PMS offset determination using an IF attenuator," ESAESTEC, Noordwijk, The Nederlands, Tech. Note 14629/00/NL/SF, Jun. 2002.

[6] M. A. Brown, F. Torres, I. Corbella, and A. Colliander, "SMOS calibration," IEEE Trans. Geosci. Remote Sens., vol. 46, no. 3, pp. 646-658, Mar. 2008.

[7] F. Torres, A. Camps, J. Bará, I. Corbella, and R. Ferrero, "On-board phase and modulus calibration of large aperture synthesis radiometers: Study applied to MIRAS," IEEE Trans. Geosci. Remote Sens., vol. GRS-34, no. 4, pp. 1000-1009, Jul. 1996.

[8] I. Corbella et al., "Calibration of the MIRAS radiometers," IEEE J. Sel. Topics Appl. Earth Observ. Remote Sens., to be published.

[9] I. Corbella, F. Torres, N. Duffo, V. González, A. Camps, and M. Vall-llossera, "Fast processing tool for SMOS data," in Proc. Int. Geosci. Remote Sens. Symp. (IGARSS), vol. 2, Boston, MA, USA, Jul. 2008, pp. 1152-1155.

[10] I. Corbella, V. González-Gambau, F. Torres, N. Duffo, I. Durán, and M. Martín-Neira, "The MIRAS 'all-licef' calibration mode," in Proc. Int. Geosci. Remote Sens. Symp. (IGARSS), Beijing, China, Jul. 2016, pp. 2013-2016. 